\title{
APPENDIX 1. CARBON AND CARBONATE ANALYSES, LEG 20
}

Gerald W. Bode, Scripps Institution of Oceanography, La Jolla, California

Leg 20 sediments were analyzed for total carbon and acid-insoluble (organic) carbon using a LECO 70 Second Analyzer. The 3-cc sediment samples were first dried at $105^{\circ}-110^{\circ} \mathrm{C}$ and then ground to a homogeneous power. The ground sediment was redried and two samples, a 0.1-gram and a 0.5 -gram sample, were then weighed into LECO clay crucibles. The 0.5-gram sample was acidified with dilute hydrochloric acid and washed with distilled water. The sample was then dried and analyzed for acid-insoluble carbon, listed in the table as "organic" carbon. The 0.1 -gram sample was analyzed for total carbon without further treatment. If the result showed less than $10 \%$ $\mathrm{CaCO}_{3}$, an additional 0.5-gram sample was analyzed for greater accuracy. The calcium carbonate percentages were calculated as follows: (\% Total C. $\%$ Organic C) $\times 8.33-\%$ $\mathrm{CaCO}_{3}$. Although other carbonates may be present, all acid-soluble carbon was calculated as calcium carbonate. All results are given in weight percent.

Precisions for the analyses are as follows:

Total carbon:

( $1.2 \%$ to $12 \%$ ): $\pm 0.2 \%$ (absolute variation)

( $0 \%$ to $1.2 \%$ ): $\pm 0.4 \%$ (absolute variation)

Organic carbon: $\pm 0.04 \%$ (absolute variation)

Calcium carbonate:

(10\% to $100 \%$ ): $\pm 2 \%$ (absolute variation)

(0\% to $10 \%$ ): $\pm 0.6 \%$ (absolute variation)

Detailed descriptions of the technique and theory may be found in Bader, Gerard, et al. (1970) and Boyce and Bode (1972).

\section{REFERENCES}

Bader, R. G., Gerard, R. D., et al., 1970. Initial Reports of the Deep Sea Drilling Project, Volume IV: Washington (U. S. Government Printing Office).

Boyce, R. E. and Bode, G. W., 1972. Carbon and carbonate analyses, Leg 9, Deep Sea Drilling Project. In Hays, J. D., et al. Initial Reports of the Deep Sea Drilling Project, Volume IX: Washington (U.S. Government Printing Office), p. 747.

TABLE 1

Carbon-Carbonate Analyses, Leg 20

\begin{tabular}{lcccc}
\hline $\begin{array}{c}\text { Core, Section } \\
\text { Top of } \\
\text { Interval } \\
(\mathrm{cm})\end{array}$ & $\begin{array}{c}\text { Depth } \\
\text { in Hole } \\
(\mathrm{m})\end{array}$ & $\begin{array}{c}\text { Carbon } \\
\text { Total } \\
(\%)\end{array}$ & $\begin{array}{c}\text { Organic } \\
\text { Carbon } \\
(\%)\end{array}$ & $\begin{array}{c}\mathrm{CaCO}_{3} \\
(\%)\end{array}$ \\
\hline Site 194 & & & & \\
$1-1,12$ & 37.6 & 0.4 & 0.2 & 1 \\
$1-3,25$ & 40.8 & 0.5 & 0.3 & 1 \\
$1-5,60$ & 44.1 & 0.2 & 0.2 & 0 \\
$1-6,56$ & 45.6 & 0.2 & 0.2 & 0 \\
$2-1,149$ & 143.5 & 0.1 & 0.1 & 1 \\
22,74 & 144.2 & 0.1 & 0.1 & 0 \\
\hline
\end{tabular}

TABLE 1 - Continued

\begin{tabular}{|c|c|c|c|c|}
\hline $\begin{array}{l}\text { Core, Section } \\
\text { Top of } \\
\text { Interval } \\
\text { (cm) }\end{array}$ & $\begin{array}{l}\text { Depth } \\
\text { in Hole } \\
(\mathrm{m})\end{array}$ & $\begin{array}{c}\text { Carbon } \\
\text { Total } \\
(\%)\end{array}$ & $\begin{array}{c}\text { Organic } \\
\text { Carbon } \\
(\%)\end{array}$ & $\begin{array}{c}\mathrm{CaCO}_{3} \\
(\%)\end{array}$ \\
\hline $\begin{array}{l}2-3,96 \\
2-4,75 \\
2-5,75\end{array}$ & $\begin{array}{l}146.0 \\
147.3 \\
148.8\end{array}$ & $\begin{array}{l}0.1 \\
0.2 \\
0.1\end{array}$ & $\begin{array}{l}0.1 \\
0.1 \\
0.1\end{array}$ & $\begin{array}{l}0 \\
1 \\
0\end{array}$ \\
\hline $\begin{array}{l}\text { Site } 195 \\
1-1,141 \\
1-2,86 \\
1-3,79 \\
1-4,87 \\
1-5,122 \\
1-6,68\end{array}$ & $\begin{array}{l}64.4 \\
65.4 \\
66.8 \\
68.4 \\
70.2 \\
71.2\end{array}$ & $\begin{array}{l}0.1 \\
0.2 \\
0.1 \\
0.1 \\
0.1 \\
0.1\end{array}$ & $\begin{array}{l}0.1 \\
0.1 \\
0.1 \\
0.1 \\
0.1 \\
0.1\end{array}$ & $\begin{array}{l}0 \\
1 \\
0 \\
0 \\
0 \\
0\end{array}$ \\
\hline $\begin{array}{l}2-1,117 \\
2-2,75 \\
2-3,75 \\
2-4,75\end{array}$ & $\begin{array}{l}121.2 \\
122.3 \\
123.8 \\
125.3\end{array}$ & $\begin{array}{l}0.1 \\
0.1 \\
0.1 \\
0.1\end{array}$ & $\begin{array}{l}0.1 \\
0.1 \\
0.1 \\
0.1\end{array}$ & $\begin{array}{l}0 \\
0 \\
0 \\
0\end{array}$ \\
\hline $\begin{array}{l}\text { Site } 196 \\
1-1,139 \\
2-1,124 \\
2-2,97 \\
2-3,51 \\
2-4,74 \\
2-5,61 \\
2-6,50\end{array}$ & $\begin{array}{r}38.9 \\
105.2 \\
106.5 \\
107.5 \\
109.2 \\
110.6 \\
112.0\end{array}$ & $\begin{array}{l}0.1 \\
0.1 \\
0.1 \\
0.1 \\
0.1 \\
0.1 \\
0.1\end{array}$ & $\begin{array}{l}0.1 \\
0.1 \\
0.1 \\
0.1 \\
0.1 \\
0.1 \\
0.1\end{array}$ & $\begin{array}{l}0 \\
0 \\
0 \\
0 \\
0 \\
0 \\
0\end{array}$ \\
\hline $\begin{array}{l}\text { Hole } 198 \mathrm{~A} \\
1-2,145 \\
1-3,28 \\
1-4,14 \\
1-6,58\end{array}$ & $\begin{array}{l}93.5 \\
93.8 \\
95.1 \\
98.6\end{array}$ & $\begin{array}{l}0.1 \\
0.1 \\
0.1 \\
0.1\end{array}$ & $\begin{array}{l}0.1 \\
0.1 \\
0.1 \\
0.1\end{array}$ & $\begin{array}{l}0 \\
0 \\
0 \\
0\end{array}$ \\
\hline $\begin{array}{l}2-1,99 \\
2-2,53 \\
3-1,50 \\
3-2,70 \\
3-3,30 \\
3-4,50 \\
3-5,50 \\
3-6,30\end{array}$ & $\begin{array}{l}101.0 \\
102.0 \\
110.0 \\
111.7 \\
112.8 \\
114.5 \\
116.0 \\
117.3\end{array}$ & $\begin{array}{l}0.1 \\
0.1 \\
0.1 \\
0.1 \\
0.1 \\
0.1 \\
0.1 \\
0.1\end{array}$ & $\begin{array}{l}0.1 \\
0.1 \\
0.1 \\
0.1 \\
0.1 \\
0.1 \\
0.1 \\
0.1\end{array}$ & $\begin{array}{l}0 \\
0 \\
0 \\
0 \\
0 \\
0 \\
0 \\
0\end{array}$ \\
\hline $\begin{array}{l}4-2,94 \\
4-3,98 \\
4-4,50 \\
4-5,40\end{array}$ & $\begin{array}{l}121.4 \\
123.0 \\
124.0 \\
125.4\end{array}$ & $\begin{array}{l}0.1 \\
0.1 \\
0.1 \\
0.1\end{array}$ & $\begin{array}{l}0.1 \\
0.1 \\
0.1 \\
0.1\end{array}$ & $\begin{array}{l}0 \\
0 \\
0 \\
0\end{array}$ \\
\hline Site 199 & & & & \\
\hline $\begin{array}{l}1-1,137 \\
1-2,36 \\
1-2,147 \\
1-3,11 \\
1-3,100 \\
1-4,26 \\
1-4,114 \\
1-5,86\end{array}$ & $\begin{array}{l}58.9 \\
59.4 \\
60.5 \\
60.6 \\
61.6 \\
62.3 \\
63.1 \\
64.4\end{array}$ & $\begin{array}{r}8.8 \\
8.9 \\
0.2 \\
8.1 \\
0.3 \\
0.4 \\
10.6 \\
0.2\end{array}$ & $\begin{array}{l}0.0 \\
0.0 \\
0.1 \\
0.0 \\
0.1 \\
0.1 \\
0.1 \\
0.1\end{array}$ & $\begin{array}{r}73 \\
73 \\
1 \\
67 \\
2 \\
3 \\
37 \\
87 \\
1\end{array}$ \\
\hline $\begin{array}{l}2-1,99 \\
2-2,100 \\
2-3,60 \\
2-4,50\end{array}$ & $\begin{array}{l}68.0 \\
69.5 \\
70.6 \\
72.0 \\
\end{array}$ & $\begin{array}{l}0.1 \\
0.1 \\
0.1 \\
0.1\end{array}$ & $\begin{array}{l}0.0 \\
0.0 \\
0.1 \\
0.0\end{array}$ & $\begin{array}{l}0 \\
1 \\
0 \\
0\end{array}$ \\
\hline
\end{tabular}


G. W. BODE

TABLE 1 - Continued

\begin{tabular}{lrrrr}
\hline $\begin{array}{c}\text { Core, Section } \\
\text { Top of } \\
\text { Interval } \\
(\mathrm{cm})\end{array}$ & $\begin{array}{c}\text { Depth } \\
\text { in Hole } \\
(\mathrm{m})\end{array}$ & $\begin{array}{c}\text { Carbon } \\
\text { Total } \\
(\%)\end{array}$ & $\begin{array}{c}\text { Organic } \\
\text { Carbon } \\
(\%)\end{array}$ & $\begin{array}{c}\mathrm{CaCO}_{3} \\
(\%)\end{array}$ \\
\hline $2-5,102$ & 74.0 & 1.9 & 0.0 & 15 \\
$2-6,45$ & 75.0 & 0.6 & 0.1 & 4 \\
& & & & \\
$3-2,106$ & 79.1 & 0.1 & 0.1 & 0 \\
$3-2,130$ & 79.3 & 0.1 & 0.1 & 0 \\
$3-3,70$ & 80.2 & 0.1 & 0.0 & 0 \\
$3-4,94$ & 81.9 & 0.4 & 0.0 & 3 \\
& & & & \\
$4-1,100$ & 87.0 & 0.2 & 0.0 & 1 \\
$4-2,75$ & 88.3 & 0.1 & 0.0 & 1 \\
$4-3,50$ & 89.5 & 0.7 & 0.0 & 5 \\
$4-4,50$ & 91.0 & 0.5 & 0.0 & 4 \\
$4-5,52$ & 92.5 & 1.9 & 0.0 & 16 \\
$4-5,102$ & 93.0 & 2.0 & 0.0 & 16 \\
\hline
\end{tabular}

TABLE 1 - Continued

\begin{tabular}{lcccc}
\hline $\begin{array}{c}\text { Core, Section } \\
\text { Top of } \\
\text { Interval } \\
(\mathrm{cm})\end{array}$ & $\begin{array}{c}\text { Depth } \\
\text { in Hole } \\
(\mathrm{m})\end{array}$ & $\begin{array}{c}\text { Carbon } \\
\text { Total } \\
(\%)\end{array}$ & $\begin{array}{c}\text { Organic } \\
\text { Carbon } \\
(\%)\end{array}$ & $\begin{array}{c}\mathrm{CaCO}_{3} \\
(\%)\end{array}$ \\
\hline & & & & \\
$5-1,93$ & 143.9 & 0.1 & 0.0 & 6 \\
$5-1,67$ & 145.2 & 0.1 & 0.0 & 0 \\
$5-3,60$ & 146.6 & 0.1 & 0.0 & 0 \\
$5-4,50$ & 148.0 & 0.1 & 0.1 & 0 \\
$5-5,73$ & 149.7 & 0.1 & 0.0 & 0 \\
$5-6,100$ & 151.5 & 0.1 & 0.0 & 0 \\
$6-1,48$ & 200.5 & 0.2 & 0.0 & 2 \\
$6-1,102$ & 201.0 & 0.9 & 0.0 & 7 \\
$6-2,30$ & 201.8 & 0.9 & 0.0 & 7 \\
$6-2,80$ & 202.3 & 0.8 & 0.0 & 6 \\
$6-2,120$ & 202.7 & 0.9 & 0.1 & 6 \\
$6-3,70$ & 203.7 & 0.5 & 0.1 & 4 \\
$6-4,84$ & 205.3 & 0.5 & 0.1 & 4 \\
$6-5,100$ & 207.0 & 0.4 & 0.0 & 3 \\
\hline
\end{tabular}

